Research Article

\title{
Ultrasonic Testing Analysis of Concrete Structure Based on S Transform
}

\author{
Juncai Xu $\mathbb{i D}^{1,2}$ and Hai Wei $\mathbb{D}^{1}$ \\ ${ }^{1}$ State Key Laboratory of Simulation and Regulation of Water Cycle, in River Basin, Beijing, China \\ ${ }^{2}$ Department of Civil Engineering, Case Western Reserve University, Cleveland, USA \\ Correspondence should be addressed to Juncai Xu; jxx307@case.edu
}

Received 2 April 2019; Revised 18 June 2019; Accepted 20 September 2019; Published 8 October 2019

Academic Editor: Ivo Caliò

Copyright ( $) 2019$ Juncai Xu and Hai Wei. This is an open access article distributed under the Creative Commons Attribution License, which permits unrestricted use, distribution, and reproduction in any medium, provided the original work is properly cited.

\begin{abstract}
Ultrasonic testing analysis is a crucial scientific component during the process and interpretation of the ultrasonic detection signal. Focusing on the ultrasonic testing characteristics, the time-invariant spectral analysis method cannot meet the processing requirements of the detection signal fully. Thus, $S$ transform, the time-varying analysis method, was introduced into the ultrasonic testing data processing of the concrete structure. The acoustic wave phase velocity was derived based on the spectrum analysis, and the $S$ transform time-frequency analysis method was established. Finally, based on the method of concrete experimental data set, studies show that the frequency energy spectrum with $S$ transform can realize flexible and effective identification of defects in the concrete structure. Definitely, this analysis method can significantly improve the resolution and practicality of ultrasonic testing.
\end{abstract}

\section{Introduction}

Applying nondestructive testing (NDT) to the concrete structure detects objects without destroying the structure using the reactions to the changes in the physical field caused by the defects in the internal structure. We can detect the defects in the interior material and surfaces to recognise defects and evaluate the quality of the concrete structure [1-3]. NDT of concrete structures is one of the critical tasks conducted in engineering quality control and management. Ultrasonic testing (UT) detection technology is a nondestructive testing technology frequently used in concrete quality management. Compared with other conventional detection technologies, UT easily penetrates deep-layered materials and accurately locates defects. It has outstanding advantages in the field [4-8].

With the rapid development of information technology, people invented various processing techniques to process ultrasonic testing signals. These techniques improve the quality of ultrasound signals, enhancing the useful signals. It extracts useful information for location and quantitation analysis of the ultrasonic signal to obtain quantitative detection [9-11]. Signal processing is one of the critical tasks in ultrasonic testing of concrete structures. It has important effects in concrete detection. Concrete ultrasonic testing signal is a wideband-modulated central frequency signal. The signal has certain frequency bandwidth, which is nonstationary. It presents the spectral information within the local time range. If we use a simple frequency or timedomain analysis method, it cannot meet the requirements for processing the detection signal. So, we need to analyse the signal considering both the time and frequency domains $[12,13]$. Thus, there are many time-frequency analysis methods proposed that are based on Fourier analysis: shorttime Fourier transform (STFT), Wigner-Ville distributions, Cohen class, $\mathrm{S}$ transform, wavelet transform (WT), and so on [14-18].

The STFT window function is fixed, and it also fixes the resolution. It only applies to the nonstationary signal analysis of the fixed bandwidth. Bilinear transforms, Wigner-Ville distribution, and Cohen class influence the cross term, which leads to the artefact phenomenon on the time-frequency plane [19]. WT has adjustable accuracy. But it does not have adaptability when the basic wavelet is 
determined. All data are analysed with the same wavelet. S transform adopts the Morlet wavelet as the basic wavelet and is extended from the substantially continuous wavelet transform. It combines the advantages of STFT and WT. Its transform result is directly related to the signal spectrum. The basic wavelet does not need to satisfy the admissible condition of the wavelet function. Compared with the Wigner-Ville distribution, Cohen class and bilinear transform, $S$ transform does not present cross terms and it has higher precision [20]. S transform has been used in many fields, such as electrical engineering, medicine, mechanical engineering, and geophysics. [21-24]. In processing the detection data of civil engineering structures, we acquire the features of the signals with mathematical transformation and obtain the defects' features. Processing the signal can effectively improve the resolution of the ultrasonic testing. Therefore, the studying of UT S transform is of great significance for the evaluation of civil engineering structures.

In this study, the ultrasonic transmission method was described in the concrete structure detection. Then, S transform theory was introduced, and the phase velocity of the ultrasound was also provided in the concrete structure. The proposed method was applied to detect the concrete structure defect testing model. The feasibility of the proposed method was analysed, and the application effect of the proposed method was verified.

\section{Materials and Methods}

2.1. Ultrasonic Detection Principle. By using the ultrasonic transmission method to detect defects in concrete, the ultrasonic propagation encounters defects in the concrete structure and then the acoustic parameters (amplitude and frequency) change in the concrete. From a comprehensive analysis of the acoustic parameters, the quality of the concrete structure can be determined (Figure 1).

The velocity of the ultrasonic in the propagating medium is an important parameter for characterizing the concrete properties. The ultrasonic velocity is also related to the wave type. In a uniform isotropic medium, the shear wave velocity $V_{T}$ is as follows:

$$
V_{T}=\sqrt{\frac{Y}{2 \rho(1+\sigma)}},
$$

where $Y$ is Young's modulus, $\sigma$ is Poisson's ratio, and $\rho$ is the density.

Similarly, the longitudinal wave velocity $V_{I}$ is as follows:

$$
V_{T}=\sqrt{\frac{Y(1-\sigma)}{2 \rho(1+\sigma)(1-2 \sigma)}} .
$$

When there are defects (void-defective concrete, cellular, cracks, etc.) in the concrete, the density, Poisson's ratio, and elastic modulus are different from those of intact concrete. The ultrasonic wave appears to reflect, refract, and diffract, which will lead to changes in the propagation velocity of the ultrasonic waves.

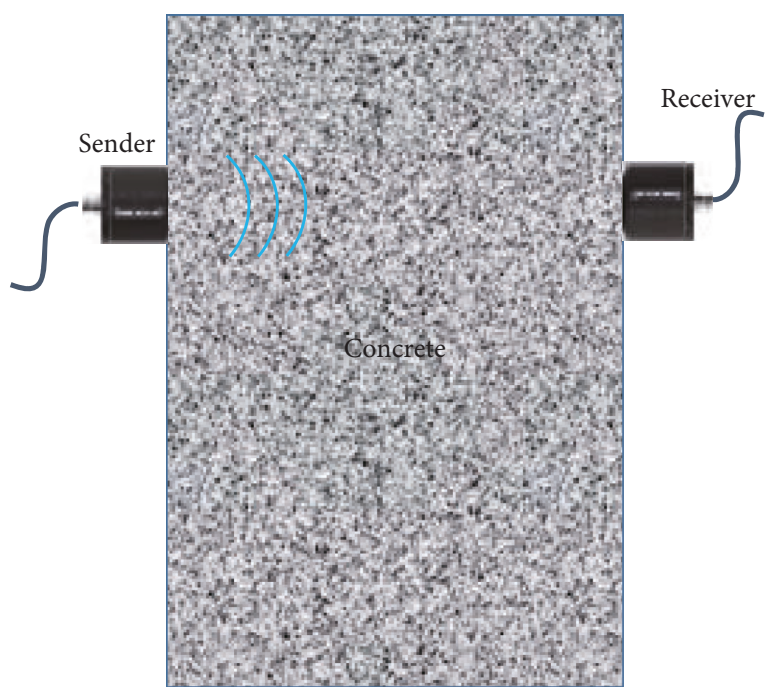

FIgURE 1: Schematic representation of experiments.

2.2. S Transform Theory. S transform combines the merits of short-time Fourier transform and WT. It regards the Morlet wavelet as the basic wavelet. Here is the formula for WT:

$$
X(a, \tau)=\frac{1}{\sqrt{a}} \int_{-\infty}^{+\infty} \psi\left(\frac{t-\tau}{a}\right) x(t) \mathrm{d} t,
$$

where $t$ denotes time, $a$ is the scale, which is the inverse of the frequency, $\tau$ is the time of the spectral localization, $x(t)$ is a function of time, and $\psi$ is the mother wavelet. The Morlet wavelet can be expressed as follows:

$$
\psi(t, f)=\frac{|f|}{\sqrt{2 \pi}} e^{i 2 \pi f t} e^{-\left(t^{2} f^{2} / 2\right)},
$$

where the frequency $f$ is the inverse of the factor $a$.

Combining equations (3) and (4), the following expression is obtained [25]:

$$
S(\tau, f)=\int_{-\infty}^{+\infty} x(t) \frac{|f|}{\sqrt{2 \pi}} e^{i 2 \pi f t} e^{-\left((\tau-t)^{2} f^{2} / 2\right)} \mathrm{d} t,
$$

with a substitution, we concluded that the $S$ transform is defined as a WT with the Morlet wavelet by the phase factor:

$$
S(\tau, f)=X(a, \tau) e^{i 2 \pi f t} .
$$

S algorithm, short-time Fourier transform, and wavelet transform are applied to analyse the transient wave signal and to determine the solution of the $\mathrm{S}$ algorithm for timevarying signal processing. One transient wave signal is Ricker wavelet whose central frequency is $100 \mathrm{~Hz}$, as shown in Figure 2.

Time-frequency of the energy spectrum can be solved by $S$ transform, short-time Fourier transform, and Wavelet transform for the transient wave signal. The energy spectrum of time-frequency $S$ transform is shown in Figure 3, the energy spectrum of time-frequency short-time Fourier transform is shown in Figure 4, and the energy spectrum of time-frequency wavelet transform is shown in Figure 5.

Based on Figures 3 5, time-frequency with $S$ transform has good solution. However, short-time Fourier transform 


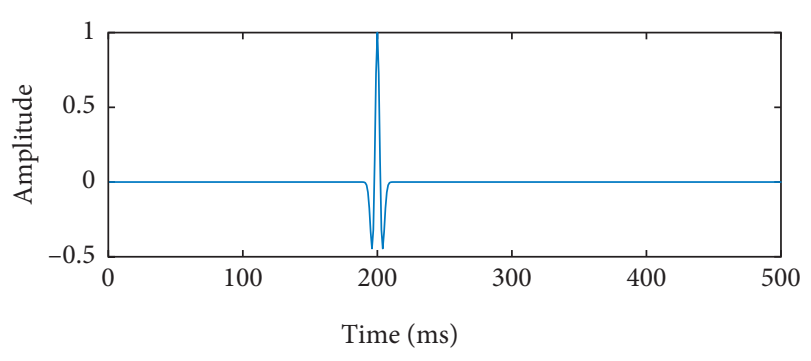

Figure 2: Ricker wavelet signal $(100 \mathrm{~Hz})$.

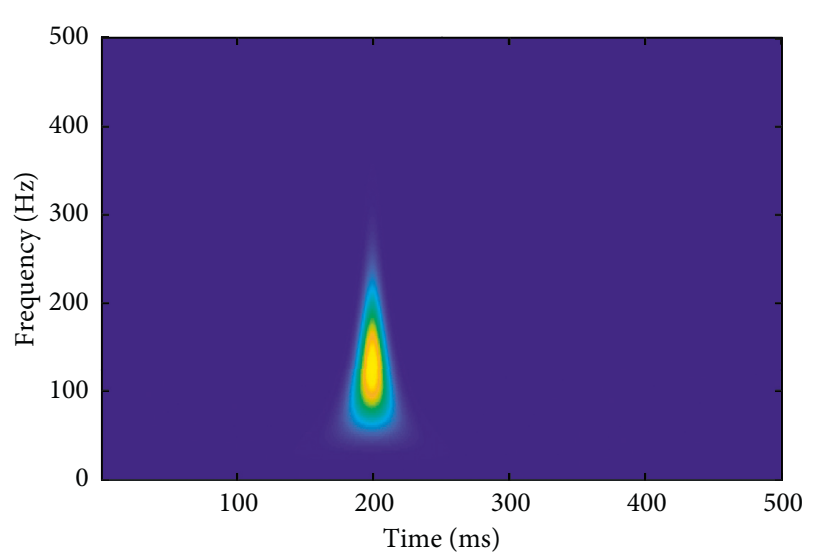

FIgURE 3: Time-frequency with S transform.

fixes the resolution, and the length of window significantly affects the solution. Time-frequency solution of wavelet transform also depends on the different wavelets. However, S transform result is directly related to the signal spectrum and does not need to set many parameters.

2.3. Phase Velocity Computation. Concrete is a dispersive material, as the ultrasound phase and group velocity are different and depend on the frequency. Phase velocities can be calculated from time-frequency diagrams [26]. The localization time of the pulse component with phase $f$ can be obtained as the first moment of energy:

$$
t_{f}=\frac{\sum_{n=M}^{N} n\left|S_{n, f}\right|^{2}}{\sum_{n=M}^{N}\left|S_{n, f}\right|^{2}} .
$$

Based on formula (7), time $t_{f}$ can be calculated for a specific frequency of a broadband pulse to cross a dispersive medium. Then, the transducer-transducer pulse can be used to compute the phase velocity:

$$
v_{f}=\frac{d}{t_{f}},
$$

where $d$ is the concrete depth.

\section{Concrete Structural Detection Experiment}

According to the specifications of the mixing ratio and the moulding process, the model specimen containing square- hole defects in the concrete was made. The model specimen had a height of $1500 \mathrm{~m}$ and a thickness of $700 \mathrm{~mm}$, so that the testing may be carried out on two symmetric sides. The four cavities' depths were $100 \mathrm{~mm}, 200 \mathrm{~mm}, 300 \mathrm{~mm}$, and $400 \mathrm{~mm}$ (Figure 6). Both specimens' surfaces were polished so that they were smooth, bright, and clean. To collect more data, survey lines were symmetrically arranged along the defect site survey line on both sides of the model specimen. From top to bottom, the three measurement lines were uniformly planned.

The ultrasonic equipment used was the Pundit PL-200 sonicator from the Swiss company Proceq SA (Figure 7). During the concrete testing process, the centre frequency of the ultrasound probe was $54 \mathrm{KHz}$, the sampling interval was $0.1 \mu \mathrm{s}$, and the sampling point number was 7,928. Based on the ultrasonic transmission detection principle, we conducted tests on the concrete specimen. Figure 8 shows the position of the detection point. The intact concrete positions were in the left side of the concrete specimens, and the positions of the $200 \mathrm{~mm}$ square holes were in the right side of the concrete specimen. The depths of the square holes were sequentially $100 \mathrm{~mm}, 300 \mathrm{~mm}, 400 \mathrm{~mm}$, and $200 \mathrm{~mm}$. The time-domain signals in the five cases of ultrasonic detection of the concrete structure were sequentially collected (Figure 9).

\section{Time-Frequency Domain Analysis Based on S Transform}

4.1. Time-Frequency Domain Analysis. First, we took the ultrasonic signal samples at the position of the intact concrete for three times. According to the $S$ transform principle, the time-domain signal was converted into the time-frequency domain energy spectrum. The results are shown in Figure 10. Thus, the $S$ transform can effectively turn the ultrasonic time-domain signal into the energy spectrum of the time-frequency domain.

Through analysis of the time-frequency domain in Figure 10, the instantaneous frequency changes can be found at three different times of the ultrasonic signal. From Figure 10, it can be seen that the instantaneous frequency keeps consistency in the three different times testing signals for the intact concrete. It can be found the experimental results are reliable. The range of the energy spectrum is from 0 to 8,000 . In the first phase, a weak energy band occurred within the range of $0 \sim 20 \mathrm{~ms}$, corresponding to a frequency within the range of $30 \sim 150 \mathrm{KHz}$. The range of time-frequency may be formed by system noise. In the energy spectrum of the time-frequency domain, one significant energy concentration area occurred within the range 160 280 ms, which was first received from the ultrasonic wave after penetrating the concrete. The time epoch for the first wave was about $160 \mathrm{~ms}$. Over time, the vibration signal gradually attenuated and a faint energy band appeared.

When studying the sample of the testing signals at the position containing the square hole, the time-domain signal was converted into the time-frequency domain energy spectrum. S transform can also turn the ultrasonic time- 


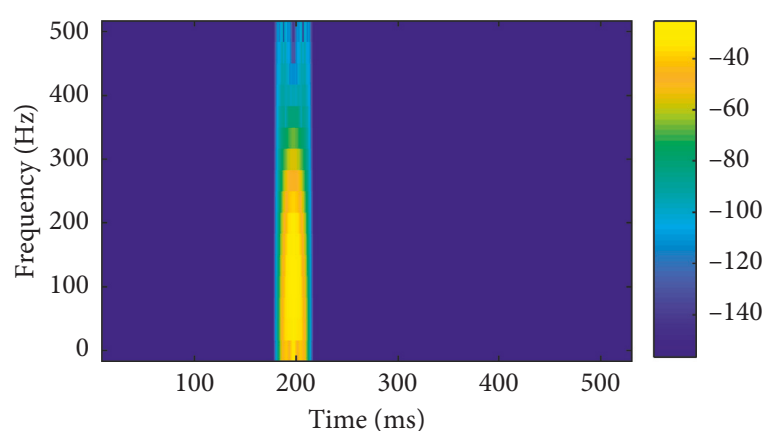

(a)

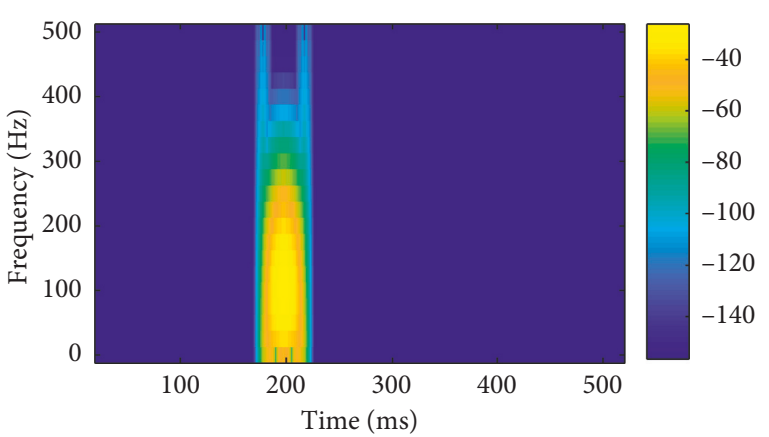

(b)

FIgURE 4: Time-frequency with short-time Fourier transform. (a) Hamming window 20 ms. (b) Hamming window 40 ms.

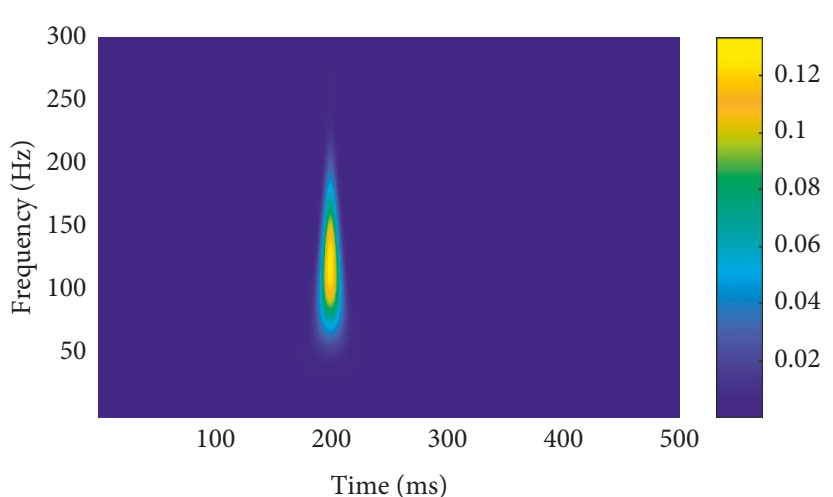

(a)

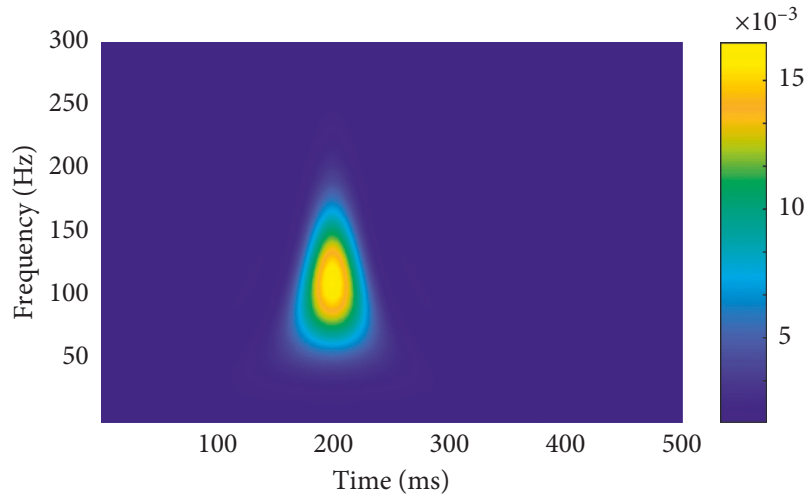

(b)

Figure 5: Time-frequency with wavelet transform. (a) Amor wavelet. (b) bump wavelet.



(a)

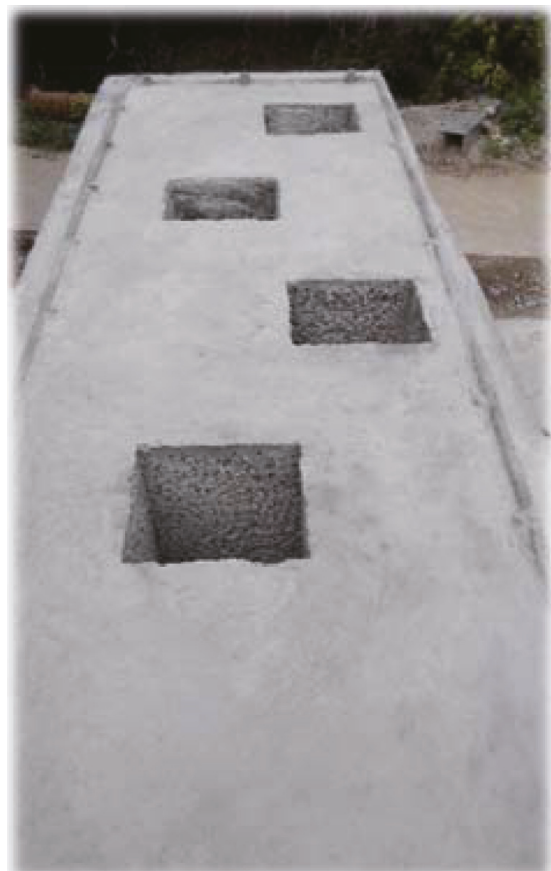

(b)

Figure 6: Concrete structure specimen construction site. (a) Pouring site. (b) Formed concrete slab. 




FIgURe 7: Pundit PL-200 sonicator.

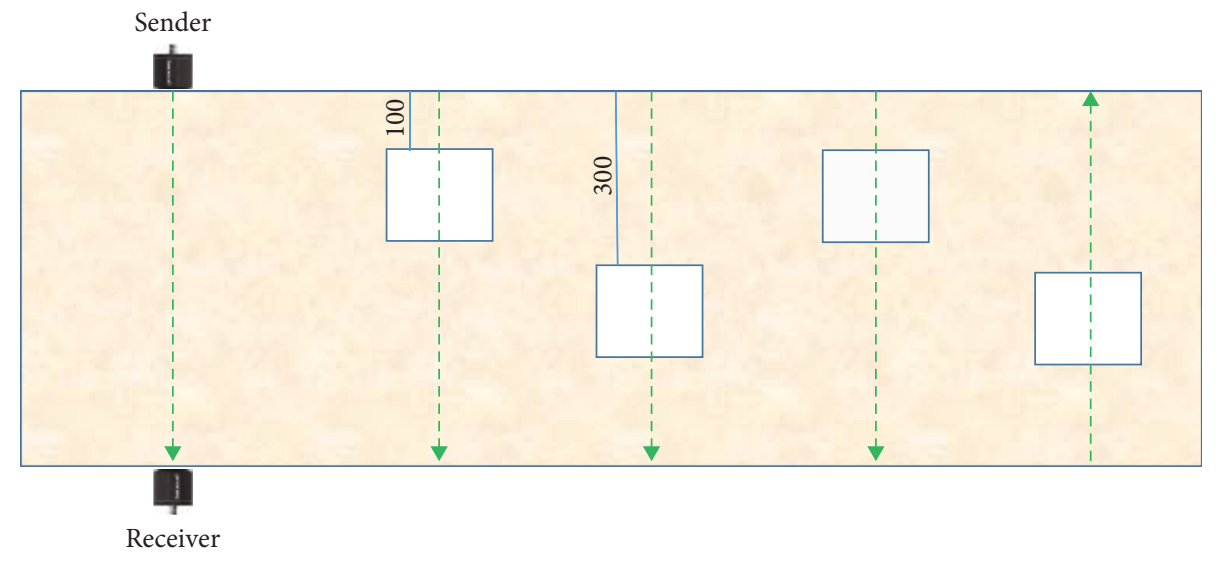

FIGURE 8: Ultrasonic testing concrete detection point schematic.

domain signal into the energy spectrum of the time-frequency domain. The results are shown in Figure 11.

Figure 11 shows the ultrasound energy spectrum at different square hole depths. The maximum of the energy spectrum reaches 3,000. In Figure 11, there is a weak energy band within the range of $0 \sim 20 \mathrm{~ms}$, corresponding to the frequency within the range of $30 \sim 150 \mathrm{KHz}$. The frequency of the interference region may have been formed by system noise. Within the time-frequency near 19 400 ms, the faint band energy also appeared, and the first wave epoch time was around $190 \mathrm{~ms}$. Then, there was one significant energy concentration area near the main frequency, forming a plurality of extreme values in the areas.

Through the comparison of Figures 10 and 11, the energy spectrum of the intact concrete structure is much higher than that of the concrete structure containing defects. When testing the intact concrete structure, there was one significant energy area near the main frequency. But when testing the concrete structure containing defects, the energy spectrum near the main frequency $50 \mathrm{KHz}$ may occur with more energy group, and the time appears to have lagged in the case of the intact concrete. When the concrete structure is in defective conditions, significant differences occur compared to the case of the intact concrete due to the acoustic refraction, reflection, diffraction, and other phenomena.

4.2. Ultrasonic Phase Velocity. In order to study the relationship between the ultrasound frequency and velocity, the corresponding relationship between the ultrasonic velocity and frequency can be calculated in the extreme region. From the time-frequency spectrum, Figure 12 shows that the ultrasonic velocity changes with the frequency in the intact concrete structure in the three different times testing signals for the intact concrete.

From Figure 12, it can be seen that the frequency-dependent velocity of the ultrasonic pulse also keeps consistency in the three different time testing signals for the intact concrete. Then the ultrasonic wave propagated in the intact concrete structure, the ultrasound velocity changed from $3,500 \mathrm{~m} / \mathrm{s}$ to $3,000 \mathrm{~m} / \mathrm{s}$ in the frequency range of $30 \sim 100 \mathrm{KHz}$. The ultrasonic wave velocity of $3,500 \mathrm{~m} / \mathrm{s}$ near the main frequency decayed to $3,000 \mathrm{~m} / \mathrm{s}$, and then, the ultrasonic velocity remained unchanged.

When there was a square hole in the concrete structure, the corresponding relationship between the ultrasonic velocity and frequency could be calculated within the extreme 


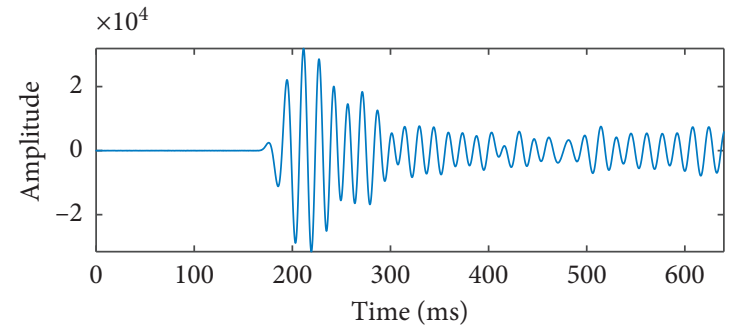

(a)

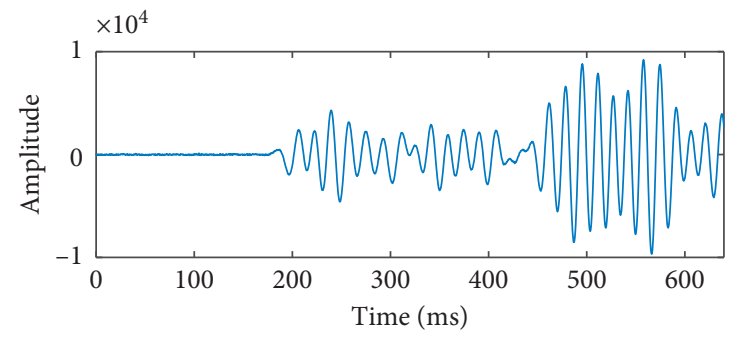

(c)

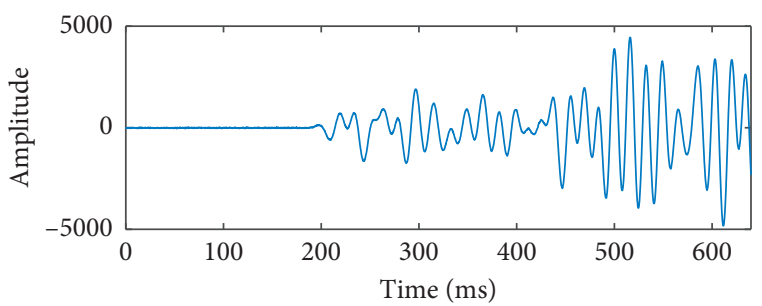

(b)

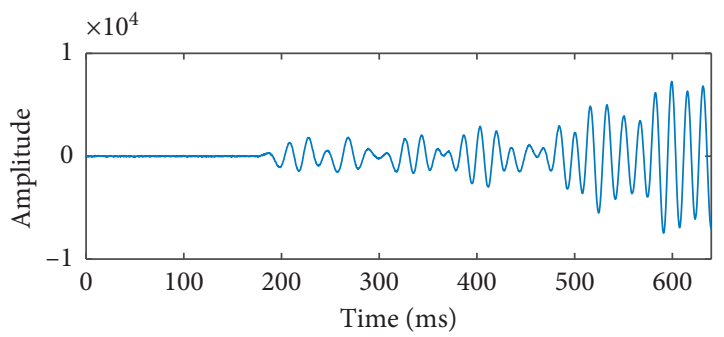

(d)

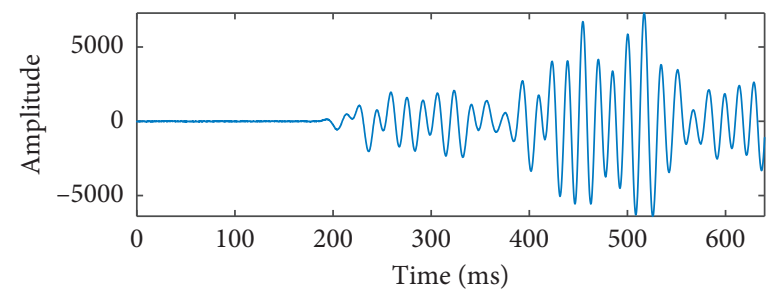

(e)

Figure 9: Time-domain ultrasonic signals in five cases: (a) intact concrete; (b) square hole depth $100 \mathrm{~mm}$; (c) square hole depth $200 \mathrm{~mm}$; (d) square hole depth $300 \mathrm{~mm}$; (e) square hole depth $400 \mathrm{~mm}$.

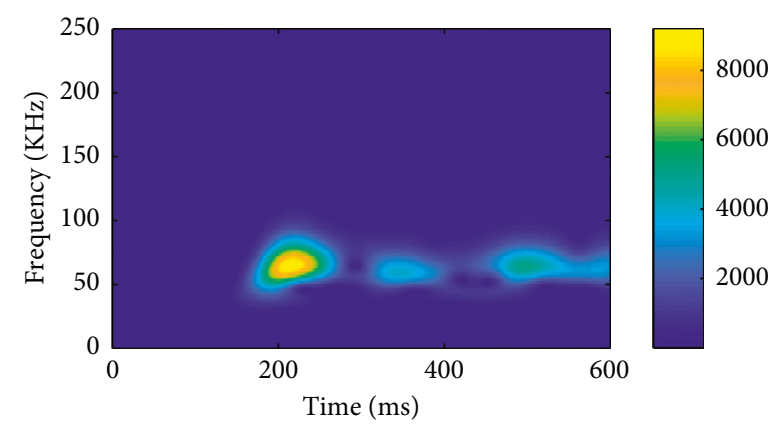

(a)

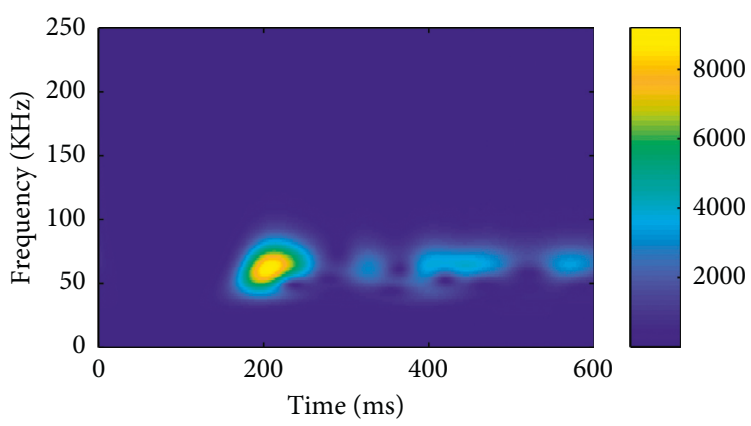

(b)

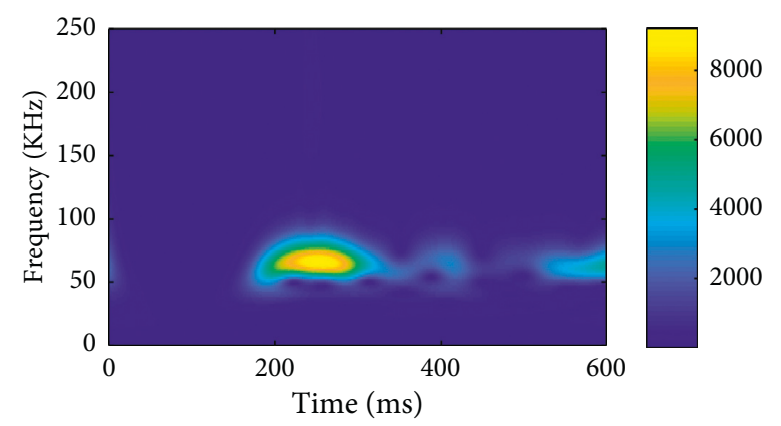

(c)

FIGURE 10: Energy spectrum of time-frequency S transform at the intact concrete position: (a) 1st testing signal; (b) 2nd testing signal; (c) 3rd testing signal. 


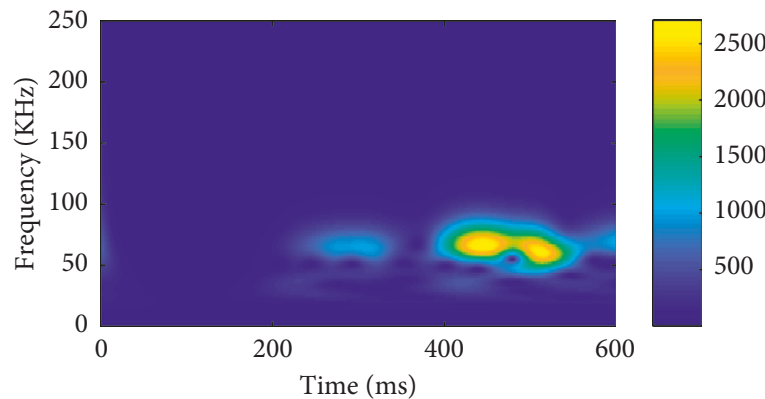

(a)

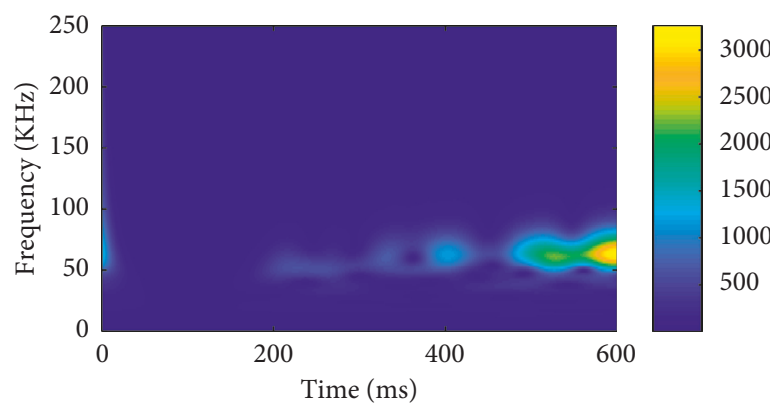

(c)

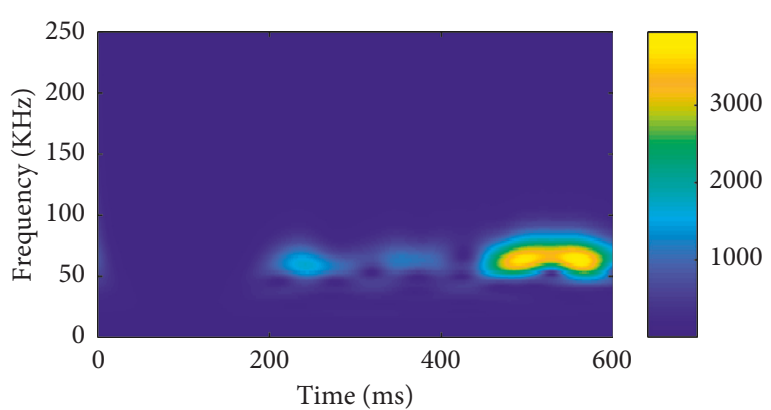

(b)

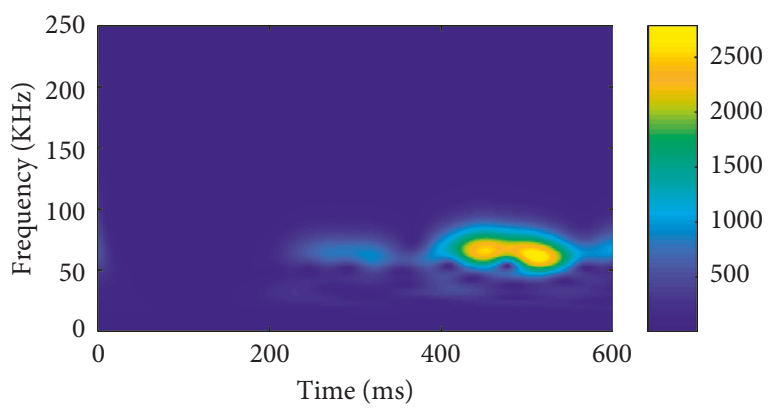

(d)

FIgURE 11: Energy spectrum of the $S$ transform: (a) square hole depth $100 \mathrm{~mm}$; (b) square hole depth $200 \mathrm{~mm}$; (c) square hole depth $300 \mathrm{~mm}$; (d) square hole depth $400 \mathrm{~mm}$.

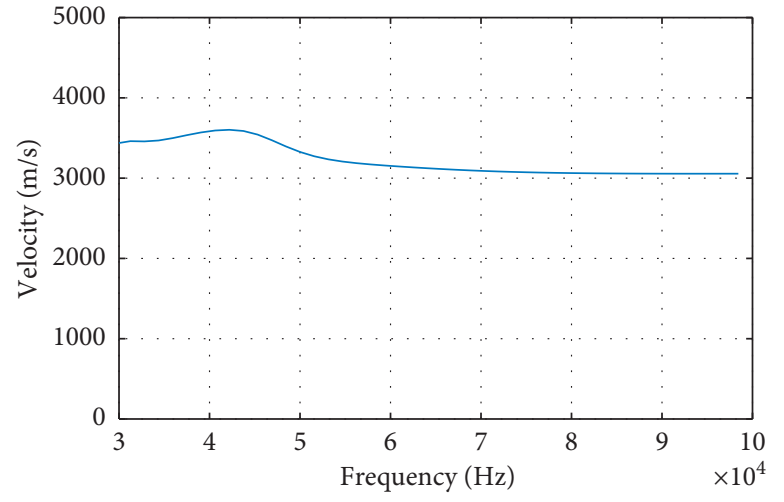

(a)

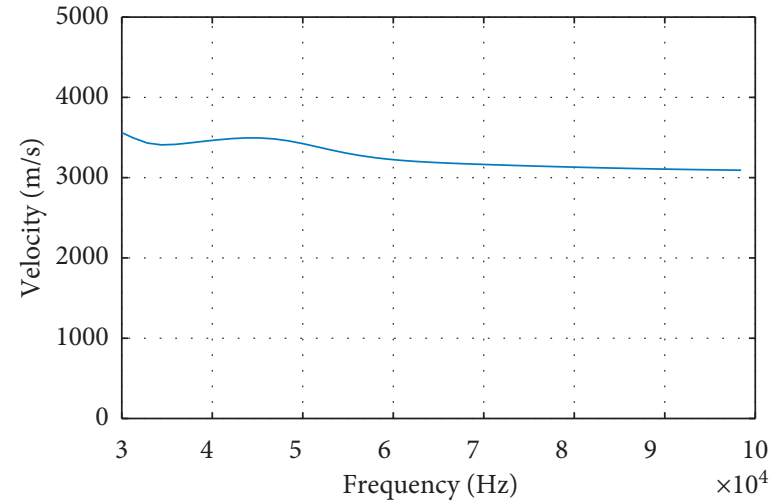

(b)

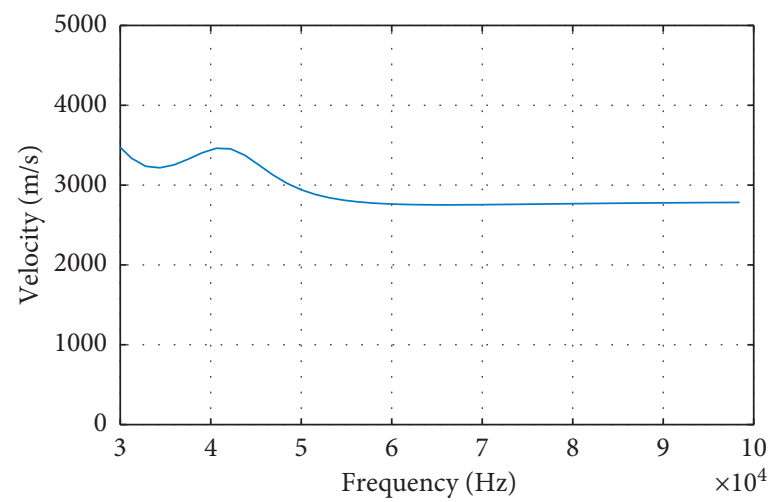

(c)

Figure 12: Frequency-dependent velocity of the ultrasonic pulse in concrete. 


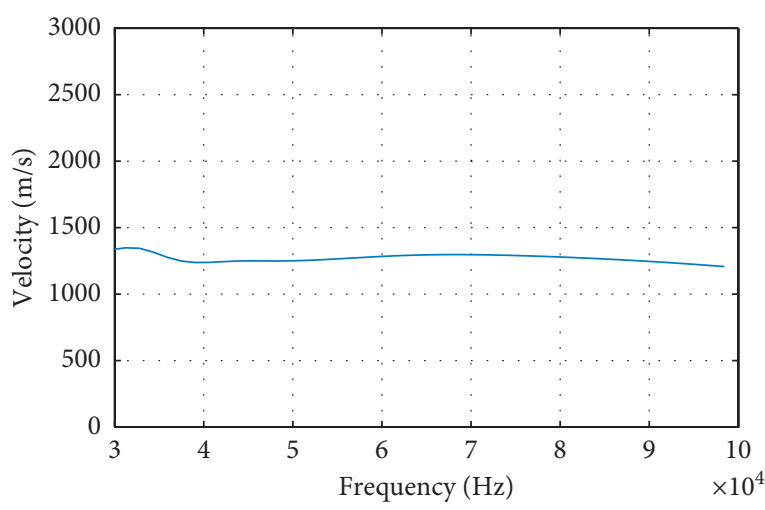

(a)

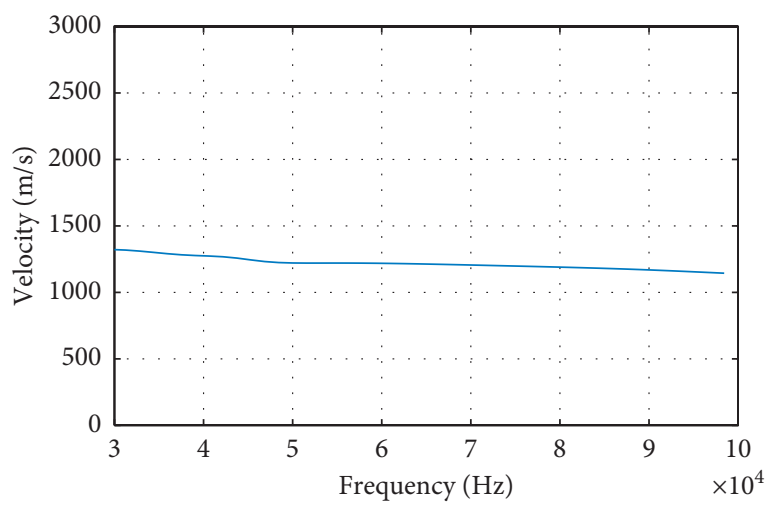

(c)



(b)



(d)

FiguRE 13: Frequency-dependent phase velocity of ultrasonic pulse in concrete: (a) square hole depth $100 \mathrm{~mm}$; (b) square hole depth $200 \mathrm{~mm}$; (c) square hole depth $300 \mathrm{~mm}$; (d) square hole depth $400 \mathrm{~mm}$.

region. Based on the frequency spectrum, Figure 13 shows the relationship between the ultrasonic frequency and velocity in the case of the defective concrete.

In Figure 13, when the ultrasonic wave propagated in the defect-filled concrete structure, the ultrasound velocity changed from $1,400 \mathrm{~m} / \mathrm{s}$ to $1,300 \mathrm{~m} / \mathrm{s}$ in the frequency range of $30 \sim 100 \mathrm{MHz}$. Then, the ultrasonic velocity remained unchanged. When comparing Figures 12 and 13, in the defective concrete structure, the extreme region velocity was much smaller than that of the intact concrete structure. Since the ultrasonic propagation velocity in air is smaller than the propagation velocity in concrete, Figure 13 shows the lower phase velocity. However, the S-transform analysis of various defects has almost the same result for all the defects. The reason may be that the ultrasonic probes used are very narrowband.

\section{Conclusions}

Based on the past concrete structure ultrasonic testing theory, a series of time-frequency analyses were carried out on ultrasound detection of concrete structures. Using the multiscale time-frequency of the $S$ transform, $S$ transform was introduced into the data processing of ultrasonic testing of the concrete structures. The ultrasonic phase velocity was derived based on the spectrum analysis. Then, $S$ transform time-frequency analysis methods were established. From the study, several conclusions can be drawn as follows:
(1) The S-transform algorithm is simple and easy to implement. It accurately transforms the ultrasonic time-domain signal into a time-frequency domain spectrum and has a significant recognition effect.

(2) In the defective concrete structure, the energy spectrum of the $S$ transform has significant differences from that of the intact concrete structure. The time-frequency energy spectrum can accurately test the presence of defects in the concrete structure based on the $\mathrm{S}$ transform.

(3) In the energy spectrum's extreme area, the ultrasonic phase velocity's change with the frequency can be calculated. In the case of the defective concrete structure, the ultrasonic phase velocity is smaller than that of the intact concrete structure.

The proposed method needs to be further applied in actual data for nondestructive detection. Future work will be conducted on improving the applicability of the method in practice.

\section{Data Availability}

The data used to support the findings of this study have not been made available because they are from other institutions and they do not permit to release the data. 


\section{Conflicts of Interest}

The authors declare that there are no conflicts of interest regarding the publication of this paper.

\section{Acknowledgments}

The research is supported by the Open Research Fund of State Key Laboratory of Simulation and Regulation of Water Cycle in River Basin (China Institute of Water Resources and Hydropower Research, grant no. IWHR-SKLKF201810) and the Open Research Fund of Key Laboratory of Hydraulic and Waterway Engineering of the Ministry of Education (Chongqing Jiaotong University, grant no. SLK2017A02).

\section{References}

[1] J. Helal, M. Sofi, and P. Mendis, "Non-destructive testing of concrete: a review of methods," Electronic Journal of Structural Engineering, vol. 14, no. 1, pp. 97-105, 2015.

[2] S. K. U. Rehman, Z. Ibrahim, S. A. Memon, and M. Jameel, "Nondestructive test methods for concrete bridges: a review," Construction and Building Materials, vol. 107, pp. 58-86, 2016.

[3] R. Pucinotti, "Reinforced concrete structure: non destructive in situ strength assessment of concrete," Construction and Building Materials, vol. 75, pp. 331-341, 2015.

[4] F. Saint-Pierre, A. Philibert, B. Giroux, and P. Rivard, "Concrete quality designation based on ultrasonic pulse velocity," Construction and Building Materials, vol. 125, pp. 1022-1027, 2016.

[5] S. Laureti, M. Ricci, M. N. I. B. Mohamed, L. Senni, L. A. J. Davis, and D. A. Hutchins, "Detection of rebars in concrete using advanced ultrasonic pulse compression techniques," Ultrasonics, vol. 85, pp. 31-38, 2018.

[6] A. Carrión, V. Genovés, J. Gosálbez, R. Miralles, and J. Payá, "Ultrasonic signal modality: a novel approach for concrete damage evaluation," Cement and Concrete Research, vol. 101, pp. 25-32, 2017.

[7] S. Iyer, S. K. Sinha, B. R. Tittmann, and M. K. Pedrick, "Ultrasonic signal processing methods for detection of defects in concrete pipes," Automation in Construction, vol. 22, pp. 135-148, 2012.

[8] Schmerr and W. Lester, Fundamentals of Ultrasonic Nondestructive Evaluation, Springer, Berlin, Germany, 2016.

[9] A. Gupta and J. C. Duke Jr., "Identifying the arrival of extensional and flexural wave modes using wavelet decomposition of ultrasonic signals," Ultrasonics, vol. 82, pp. 261-271, 2018.

[10] K. A. Tiwari, R. Raisutis, and V. Samaitis, "Signal processing methods to improve the signal-to-noise ratio (snr) in ultrasonic non-destructive testing of wind turbine blade," Procedia Structural Integrity, vol. 5, pp. 1184-1191, 2017.

[11] A. Abudhahir, K. Shankar, and S. Pandi, "Detection and classification of discontinuities using discrete wavelet transform and mfl testing," Materials Evaluation, vol. 76, no. 6, pp. 706-715, 2018.

[12] C. L. Nogueira, "Wavelet analysis of ultrasonic pulses in cement-based materials," ACI Materials Journal, vol. 107, no. 3, p. 248, 2010.

[13] E. H. Saenger, G. Karl Kocur, R. Jud, and M. Torrilhon, "Application of time reverse modeling on ultrasonic non- destructive testing of concrete," Applied Mathematical Modelling, vol. 35, no. 2, pp. 807-816, 2011.

[14] M. Le, J. Kim, S. Kim, and J. Lee, "B-scan ultrasonic testing of rivets in multilayer structures based on short-time fourier transform analysis," Measurement, vol. 128, pp. 495-503, 2018.

[15] W. Zeng, H. Wang, G. Tian, and G. Hu, "Application of laser ultrasound imaging technology in the frequency domain based on Wigner-Ville algorithm for detecting defect," Optics \& Laser Technology, vol. 74, pp. 72-78, 2015.

[16] A. Mostavi, N. Kamali, N. Tehrani, S.-W. Chi, D. Ozevin, and J. E. Indacochea, "Wavelet based harmonics decomposition of ultrasonic signal in assessment of plastic strain in aluminum," Measurement, vol. 106, pp. 66-78, 2017.

[17] C. Q. Gómez Muñoz, A. Arcos Jiménez, and F. P. García Márquez, "Wavelet transforms and pattern recognition on ultrasonic guides waves for frozen surface state diagnosis," Renewable Energy, vol. 116, pp. 42-54, 2018.

[18] W. Liu, S. Cao, and Y. Chen, "Seismic time-frequency analysis via empirical wavelet transform," IEEE Geoscience and Remote Sensing Letters, vol. 13, no. 1, pp. 28-32, 2016.

[19] R. B. Pachori, A. Nishad, and A. Nishad, "Cross-terms reduction in the Wigner-Ville distribution using tunable-Q wavelet transform," Signal Processing, vol. 120, pp. 288-304, 2016.

[20] L. Wu and J. Castagna, "S-transform and fourier transform frequency spectra of broadband seismic signals," Geophysics, vol. 82, no. 5, pp. 71-81, 2017.

[21] R. Kumar, B. Singh, D. T. Shahani, A. Chandra, and K. AlHaddad, "Recognition of power-quality disturbances using S-Transform-based ann classifier and rule-based decision tree," IEEE Transactions on Industry Applications, vol. 51, no. 2, pp. 1249-1258, 2015.

[22] S. Ari, M. K. Das, and A. Chacko, "ECG signal enhancement using S-transform," Computers in Biology and Medicine, vol. 43, no. 6, pp. 649-660, 2013.

[23] M. Manap, A. R. Abdullah, N. Z. Saharuddin, N. A. Abidullah, N. S. Ahmad, and M. H. Jopri, "Performance comparison of vsi switches faults analysis using stft and S transform," Applied Mechanics and Materials, vol. 785, pp. 210-214, 2015.

[24] Z.-l. Huang, J. Zhang, T.-h. Zhao, and Y. Sun, "Synchrosqueezing S-transform and its application in seismic spectral decomposition," IEEE Transactions on Geoscience and Remote Sensing, vol. 54, no. 2, pp. 817-825, 2016.

[25] R. G. Stockwell, L. Mansinha, and R. P. Lowe, "Localization of the complex spectrum: the S transform," IEEE Transactions on Signal Processing, vol. 44, no. 4, pp. 998-1001, 1996.

[26] C. L. Nogueira, "Wavelet-based analysis of ultrasonic longitudinal and transverse pulses in cement-based materials," Cement and Concrete Research, vol. 41, no. 11, pp. 1185-1195, 2011. 


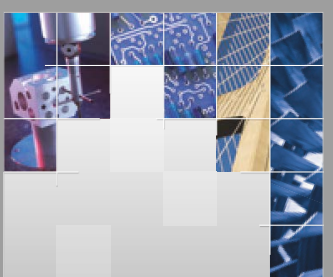

\section{Enfincering}
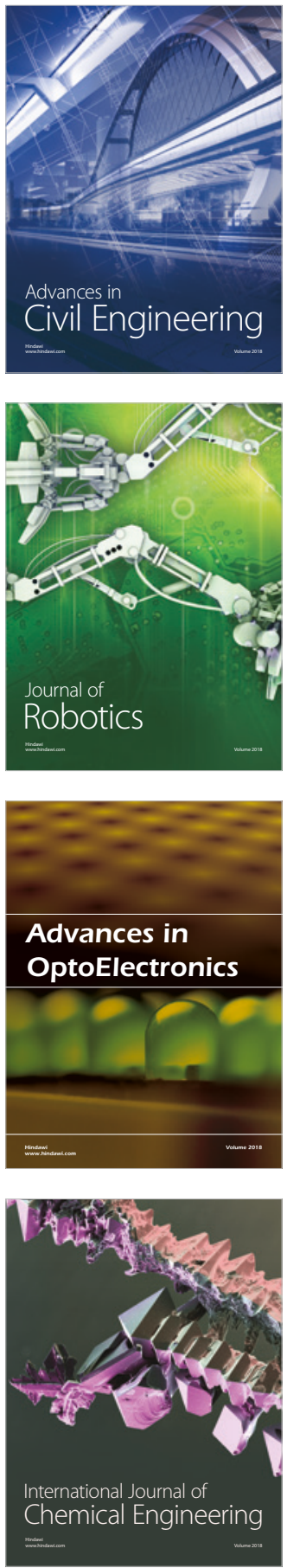



\section{Rotating \\ Machinery}

The Scientific World Journal

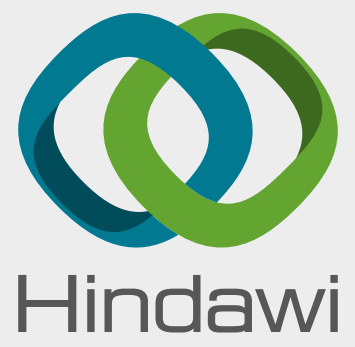

Submit your manuscripts at

www.hindawi.com

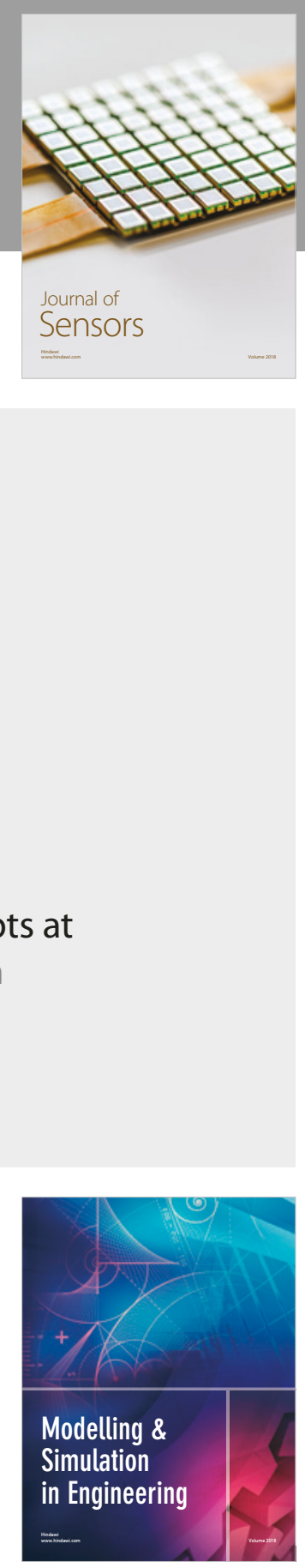

\section{Advances \\ Multimedia}
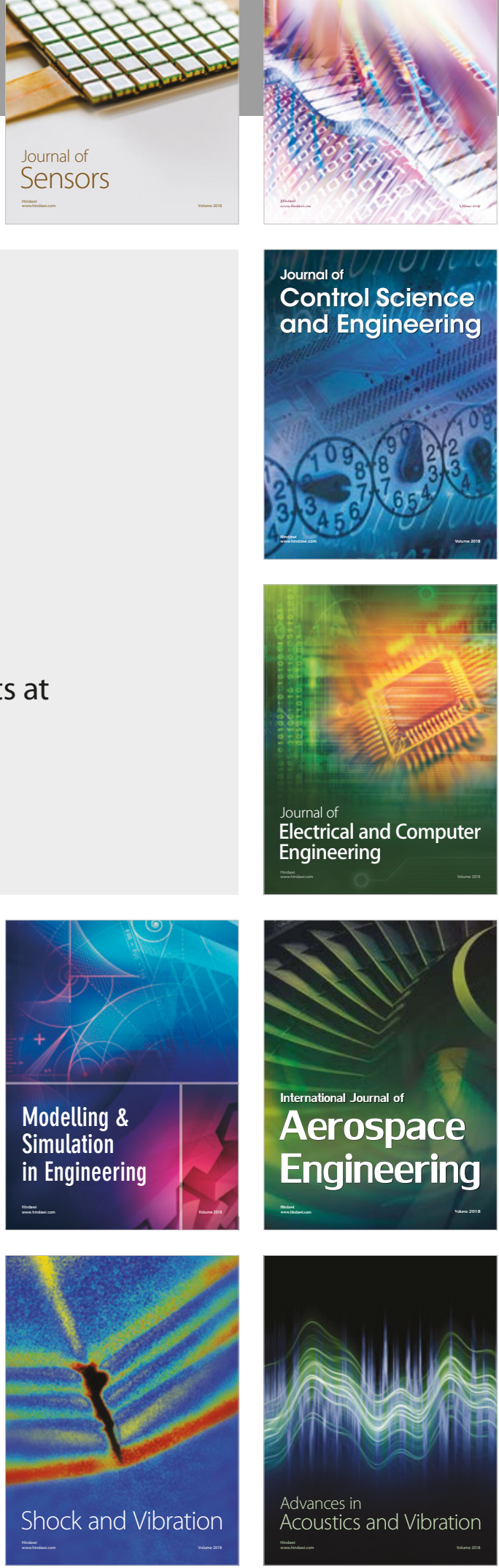